\title{
White matter fractional anisotropy predicts balance performance in older adults
}

\author{
Annouchka Van Impe ${ }^{\mathrm{a}}$, James P. Coxon ${ }^{\mathrm{a}}$, Daniel J. Goble ${ }^{\mathrm{a}}$, Mihail Doumas ${ }^{\mathrm{b}}$, \\ Stephan P. Swinnen ${ }^{\mathrm{a}, *}$ \\ ${ }^{a}$ Research Center for Movement Control and Neuroplasticity, Department of Biomedical Kinesiology, K.U. Leuven, Heverlee, Belgium \\ ${ }^{b}$ Experimental Psychology Laboratory, Department of Psychology, K.U. Leuven, Leuven, Belgium
}

Received 7 October 2010; received in revised form 14 June 2011; accepted 17 June 2011

\begin{abstract}
Aging is characterized by brain structural changes that may compromise motor functions. In the context of postural control, white matter integrity is crucial for the efficient transfer of visual, proprioceptive and vestibular feedback in the brain. To determine the role of age-related white matter decline as a function of the sensory feedback necessary to correct posture, we acquired diffusion weighted images in young and old subjects. A force platform was used to measure changes in body posture under conditions of compromised proprioceptive and/or visual feedback. In the young group, no significant brain structure-balance relations were found. In the elderly however, the integrity of a cluster in the frontal forceps explained $21 \%$ of the variance in postural control when proprioceptive information was compromised. Additionally, when only the vestibular system supplied reliable information, the occipital forceps was the best predictor of balance performance (42\%). Age-related white matter decline may thus be predictive of balance performance in the elderly when sensory systems start to degrade.
\end{abstract}

(C) 2011 Elsevier Inc. All rights reserved.

Keywords: Aging; Diffusion tensor imaging (DTI); White matter; Postural control

\section{Introduction}

In elderly individuals postural control gradually declines (Cohen et al., 1996; Horak et al., 1989; Maki et al., 1999; Stelmach et al., 1989), leading to an increasing risk of falls and so-called postfall syndrome, i.e., decreased mobility and increased rigidity due to fear of falling. Apart from musculoskeletal changes (Lord et al., 1991), postural control in the elderly declines as a result of reduced neural integration of visual, vestibular, and proprioceptive feedback, leading to inappropriate or suboptimal balance motor commands. With respect to proprioceptive feedback, research has already shown that the elderly have reduced

\footnotetext{
* Corresponding author at: Laboratory of Motor Control, Research Center for Movement Control and Neuroplasticity, Group Biomedical Sciences, K.U. Leuven, Tervuursevest 101, B-3001 Heverlee, Belgium. Tel.: +32163290 71; fax: +3216329197.

E-mail address: Stephan.Swinnen@faber.kuleuven.be (S.P. Swinnen).
}

numbers of cutaneous (Maki et al., 1999) and joint mechanoreceptors (Aydoğ et al., 2006), contributing to a reduced joint position sense (Goble et al., 2009). Visual feedback may be compromised because the eyes are subject to agerelated pathologies (cataract, glaucoma, etc.) (Leibowitz et al., 1980) and furthermore, the vestibular system is impaired in approximately $30 \%$ of the people over the age of 70 (Fife and Baloh, 1993). In addition to these peripheral changes, efficient integration of different sensory inputs in the brain might be compromised because of age-related decline in gray (Good et al., 2001; Kalpouzos et al., 2009; Smith et al., 2007) and white matter integrity (Abe et al., 2002; Barrick et al., 2010; Nusbaum et al., 2001; Sullivan and Pfefferbaum, 2006, 2007; Sullivan et al., 2001; Zhang et al., 2010).

During the past decade the effect of brain aging on postural control has gained increasing interest from neuroscientists using medical imaging. Guttmann et al. (2000) were 1 of the first reporting reduced whole white matter 
volume in so-called mobility-impaired elderly. Later on, region of interest (ROI)-analyses were performed to refine the study of white matter changes. Sullivan et al. (2001) acquired diffusion weighted scans from a sample encompassing the adult life-span and correlated fractional anisotropy (FA), a measure of white matter integrity, with scores from an ataxia test battery. The time spent standing on $1 \mathrm{leg}$ with eyes closed appeared to be positively correlated with the FA within 4 ROIs: genu and splenium of the corpus callosum, centrum semiovale, and parietal pericallosal area. In a later study by the same group (Sullivan et al., 2010), only the FA of the frontal forceps was reported to show a positive correlation with this measure. However, additional multiple regression analyses revealed that age, and not the integrity of the frontal forceps, was the predominant factor driving this relationship.

A complementary study (Sullivan et al., 2009) was performed in which fluid attenuated inversion recovery (FLAIR) images were acquired. White matter hyperintensity burden, an index of fluid accumulation as a result of white matter lesions, was calculated and correlated to postural sway (as measured on a force platform). Greater postural sway was found to be related to greater supratentorial cerebrospinal fluid volume in men, and higher white matter hyperintensity burden in women. Unfortunately this analysis did not allow identifying the potential role of normal-appearing white matter in postural sway, and comparison with previous studies is thus difficult. Ryberg et al. (2007) applied a similar approach, using balance scores on a semi- and fulltandem stance test. They acquired fluid attenuated inversion recovery images in order to measure the volume of $5 \mathrm{sub}$ regions of the corpus callosum. None of these volumes were significantly correlated with balance performance.

In view of these contradicting results more research is needed to further clarify the role of white matter integrity in postural control of healthy elderly. Exploring such relations between brain structure and behavior may indirectly contribute to the development of therapies that target central processing in order to improve postural control in elderly, and prolong independent living. The present study elaborates on previous results by testing upright stance in old and young subjects under dynamic conditions, while compromising visual as well as proprioceptive feedback. In contrast to many balance test batteries that are aimed at detecting loss of balance, the dynamic posturography setup used here was developed to identify how subjects reweight sensory information in order to maintain balance control (Black et al., 1983; Goebel et al., 1997; Monsell et al., 1997; Nashner et al., 1982). By systematically degrading sensory feedback, it provides a more complete picture of postural control in elderly.

A voxel-based analysis of the white matter was performed in order to detect balance-brain structure relations that might have been overlooked by previous studies using a ROI-based approach. Tract based spatial statistics (TBSS) was used to detect age-related white matter changes on a voxelized skeleton, representing the center of white matter tracts. A regression approach was then applied in both age groups to detect white matter regions that are correlated with postural control. Based on previous work underscoring the need for age-related increased cognitive control over motor performance in general (Heuninckx et al., 2005, 2008; Swinnen et al., 2010) and balance in particular (Dault et al., 2001a, 2001b; Doumas et al., 2009; Huxhold et al., 2006; Rankin et al., 2000), we speculated that especially the integrity of the frontal white matter tracts would be predictive of the older subjects' balance performance.

\section{Methods}

\subsection{Subjects}

Thirty-one young adults (17 female, mean age 25.3, years, range 19.8-32.4) and 36 older adults (18 females, mean age, 68.6; range 62.0-81.1) with no history of neurological diseases participated in the study. Scans from 2 older subjects contained artifacts and were therefore discarded from all further analyses. All participants had normal or corrected to normal vision and were right-handed, as assessed by the Edinburgh Handedness Inventory (Oldfield, 1971). The Mini-Mental State Examination (Folstein et al., 1975) was used to determine general cognitive function. All the elderly scored within normal limits (score $\geq 27$ ). Participants were informed about the experimental procedures and provided written informed consent. The study was approved by the local Ethics Committee of K.U. Leuven and was performed in accordance with the 1964 Declaration of Helsinki.

\subsection{Experimental design}

\subsubsection{Sensory organization test}

Two or 3 days prior to scanning, postural control was tested on an Equitest balance platform (Neurocom International, Inc., Clackamas, OR, USA). This dynamic postural test system consists of a surface with dual forceplate $(23 \times$ $46 \mathrm{~cm}$ ), force transducers and a movable visual surround. The Sensory Organization Test (SOT) was administered, whereby the forceplate measures the angular displacement $(\theta)$ of the center of gravity (COG) of the subject's body, and under certain conditions (see further), adjusts the surface concurrently so as to maintain a constant ankle joint position (sway-referencing). We used the SOT to measure subjects' reliance on different sensory systems and their ability to correct posture under compromised conditions (Doumas and Krampe, 2010). Subjects stood on the surface barefoot, with the medial malleoli of the ankles aligned to the centers of rotation of the forceplate. A safety harness was used to prevent falls in case of loss of balance. Four conditions were of interest in which visual and proprioceptive feedback were systematically altered (Fig. 1). 

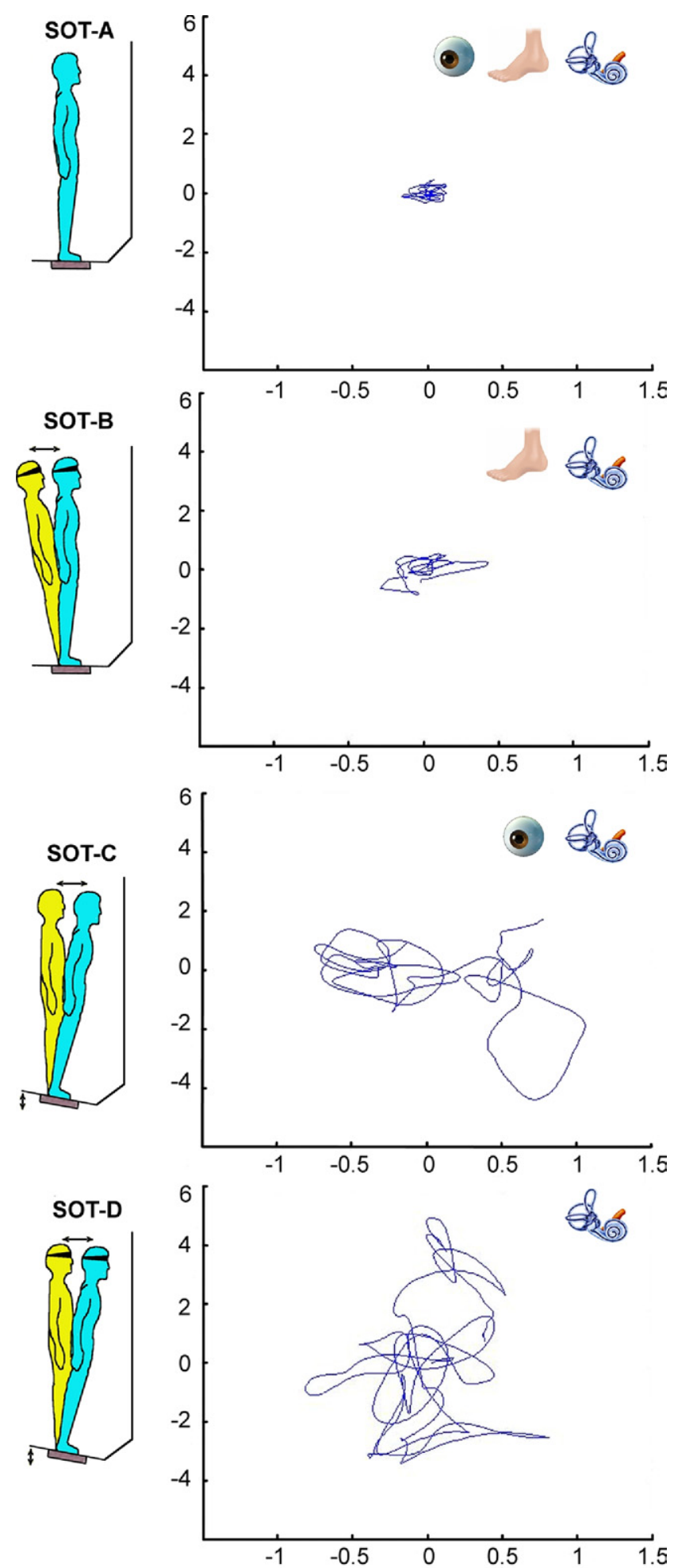

Fig. 1. Schematic representation of the 4 conditions of the Sensory Organization Test and an example data set of an old subject's center of pressure (COP) displacement $(\mathrm{cm})$ in anterior-posterior (AP) (y-axis) and mediallateral (ML) (x-axis) direction across a 20-second trial. In the top right corner of the graphs the available sensory systems (visual, proprioceptive, or vestibular) are indicated. Adapted with the permission of Neurocom International, Inc., Clackamas, OR, USA.
SOT-A required upright stance in standard conditions, where visual, proprioceptive and vestibular feedback were available to control posture. SOT-B was the same, except that the subject was asked to close his/her eyes, thereby eliminating all visual information. In SOT-C, the subject's eyes were open and the surface was sway-referenced, thereby keeping the ankle joint position constant. The resulting ankle proprioceptive feedback was thus no longer a useful indicator of body sway, and therefore a reweighing of sensory inputs was required. Finally, in SOT-D the surface was again sway-referenced and the subject was now asked to close his/her eyes. Hence only the vestibular system provided accurate feedback about body position. Each condition was repeated 3 times, resulting in 12 trials. A trial lasted 20 seconds. Subjects always started by performing SOT-A, whereas the order of the remaining trials was randomized within subjects.

\subsubsection{Image acquisition protocol}

All scanning was performed on a Siemens 3 T Magnetom Trio MRI scanner (Siemens, Erlangen, Germany) with 12 channel matrix head coil, using a diffusion tensor imaging (DTI) SE-EPI (diffusion weighted single shot spin-echo echoplanar imaging) sequence (repetition time $=7200$; echo time $=81 \mathrm{~ms}$; matrix $=96 \times 96,2.86 \times 2.18 \times 2.18$ $\mathrm{mm}$ voxels, 56 sagittal slices). Diffusion sensitizing gradients were applied at a b-value of 1000 seconds $/ \mathrm{mm}^{2}$, along 64 noncollinear directions. One b0 image with no diffusion weighting was acquired. A 3D magnetization prepared rapid acquisition gradient echo (MPRAGE) high resolution T1weighted image (repetition time $=2300 \mathrm{~ms}$, echo time $=$ $2.98 \mathrm{~ms}$, field of view $=240 \times 256,1 \times 1 \times 1.1 \mathrm{~mm}$ voxels, 160 sagittal slices) was acquired to check for possible abnormalities in gray matter.

\subsection{Data analysis}

\subsubsection{Postural control}

Custom-written Matlab scripts (Matlab 7.4, MathWorks, Natick, MA, USA) were used to calculate the anteriorposterior (AP) sway and medial-lateral (ML) sway, based on the center of pressure (COP). The AP and ML components of the COP trajectory were first low-pass filtered, using a fourth order Butterworth dual-pass filter (cutoff frequency: $10 \mathrm{~Hz}$ ). Then an $88 \%$ confidence ellipse was fitted onto the $x-y$ plane of the COP trajectory using principal-component analysis, thereby excluding outliers. The length of the ellipse axes was equal to 2 standard deviations of the COP trajectory along each axis. Increase in the size of the ellipse area reflects a decrease in postural stability. For details on this method see Duarte and Zatsiorsky (2002) and Oliveira et al. (1996). Additionally an equilibrium score (Eq) was calculated according to the following formula:

$$
\mathrm{Eq}=[12.5-(\theta \max -\theta \min )] \times 100 / 12.5^{\circ}
$$

whereby $\theta$ is the angular displacement of the center of gravity of the subject in the AP direction. This score compares sub- 
jects' AP sway to a theoretical sway stability limit of $12.5^{\circ}$, i.e., bigger sway angles will lead to falling (Brouwer et al., 1998). A lower Eq is interpreted as more sway; a fall or adjustment of the feet to prevent falling was scored as 0 . The ellipse areas and Eq were subjected to analysis of variance (ANOVA) for repeated measurements with between-subjects factor "age group" (young and old) and the within-subjects factors "proprioceptive feedback" (2 levels: normal or sway-referenced) and "visual feedback" (2 levels: normal or absent). The level of significance was set to $\alpha=0.05$.

\subsubsection{DTI: preprocessing}

TBSS (Smith et al., 2006), part of the FSL software package (Smith et al., 2004) (Functional MRI of the Brain Software Library (FMRIB), Oxford, UK), was used for a voxel-based analysis of the DTI data. Scans of all subjects were first skull-stripped using the Brain Extraction Tool. The data were then corrected for subject motion and eddycurrent induced geometric distortions (Jones and Basser, 2004). Subsequently the diffusion sensitizing gradients ("bvecs") were rotated to correct for motion and the diffusion tensor model was fit to the data, from which FA-images were calculated. In order to correct for morphological individual differences, scans were registered to a common space (FA158 template in Montreal Neurological Institute (MNI) space, www.fmrib.ox.ac.uk/fsl/data/FMRIB58_FA.html), using FMRIB's Non-Linear Image Registration Tool (FNIRT). Next a mean FA image was created which was then eroded to a skeleton, representing the medial trajectory of white matter tracts. This group-average skeleton was thresholded at FA $>0.25$. Last, scans of all subjects were projected onto this group-average skeleton. Tbss_non_FA was used to apply the nonlinear registration and skeletonization to the axial diffusivity (the first eigenvalue) and the radial diffusivity (the mean of the second and third eigenvalue) images.

\subsubsection{DTI: statistical analysis}

Randomized permutation testing (10,000 permutations) was used for statistical inference (Nichols and Holmes, 2002). First a $t$-test was performed to detect clusters of voxels for which FA-values were significantly smaller in the old as compared with the young group, i.e., regions that were subject to age-related white matter decline. The mean FA-skeleton was used as an inclusive mask. Cluster-based thresholding by using the null distribution of the maximal cluster-mass $(t=3.1, p<0.05$, family wise error [FWE] corrected) was used to detect significant white matter clusters. Secondly a regression approach was used to identify voxels for which FA values showed a linear trend with the previously discussed Eq balance metric. There were 6 explanatory variables in the design. The first 2 explanatory variables specified the groups. The following 2 explanatory variables contained the demeaned ages for old and young subjects respectively. Because the age range in the group of elderly comprised 19 years, this factor was entered into the design as a confounding variable to be able to characterize
DTI-balance correlations that were attributed to white matter integrity alone. The remaining 2 explanatory variables specified the demeaned Eq. Because ellipse area and Eq were correlated $\left(r_{\text {young }}=-0.61\right.$, and $r_{\text {old }}=-0.74$, averaged across the 4 conditions, all $p$-values $<0.05$ ), running the analysis with ellipse area instead of Eq provided similar results. Therefore only the results of the Eq analysis are reported. The analysis was performed for each condition separately. For all statistical maps threshold-free cluster enhancement (TFCE) (Smith and Nichols, 2009) was used to identify significant white matter clusters $(p<0.05$, FWEcorrected). Alternatively, a more stringent cluster-based thresholding by using the null distribution of the maximal cluster-mass ( $t=3.1, p<0.05$, FWE-corrected) was used, to further distinguish the contribution of certain white matter tracts to balance performance. For each subject the mean FA value was extracted from the observed clusters, and these data were subjected to a stepwise linear regression (Matlab 7.4; Mathworks) in order to plot the regression line and obtain adjusted $R^{2}$ values. The regression model thus contained Eq as a dependent variable, and age and mean FA of the observed region as independent predictors.

To determine whether the observed FA-Eq relations were attributed to axial or to radial diffusivity, the above analysis was repeated for these measures. The Johns Hopkins University atlas (fsl.fmrib.ox.ac.uk/fsl/fslview/atlas-descriptions.html), as supplied by FSL, was used to localize significant voxels.

\section{Results}

\subsection{Postural control}

Balance performance during each condition was transformed into an Eq metric and the corresponding ellipse area was calculated. For ellipse area both age groups contained an outlier (more that $3 \mathrm{SD}$ from the mean). Data from these 2 subjects were therefore removed from the analysis. For both $\mathrm{Eq}$ and ellipse area, repeated measures analysis of variance showed a significant main effect of group (Eq: $F(1,63)=19.17, p<0.0001$, ellipse area: $F(1,61)=8.10$, $p<0.05)$, proprioceptive feedback $(\mathrm{Eq}: F(1,63)=302.34$, $p<0.0001$, ellipse area: $F(1,61)=11.2, p<0.0001)$ and visual feedback (Eq: $F(1,63)=205.25, p<0.0001$, ellipse area: $F(1,61)=75.5, p<0.001)$, i.e., balance performance was worse (lower Eq and bigger ellipse area) in the old versus the young group, when proprioceptive feedback was sway-referenced versus normal and when vision was absent versus normal. Furthermore there was a significant "proprioceptive feedback $\times$ visual feedback $\times$ age group" interaction for both metrics (Eq: $F(1,63)=5.78, p<0.05$, ellipse area: $F(1,61)=4.4, p<0.05)$. The interaction shown in Fig. 2 can be interpreted as follows: compared with upright stance in stable conditions (SOT-A), both groups showed more sway when proprioceptive feedback was compromised (SOT-C), and sway additionally increased when vision was absent (SOT-D). Although the 


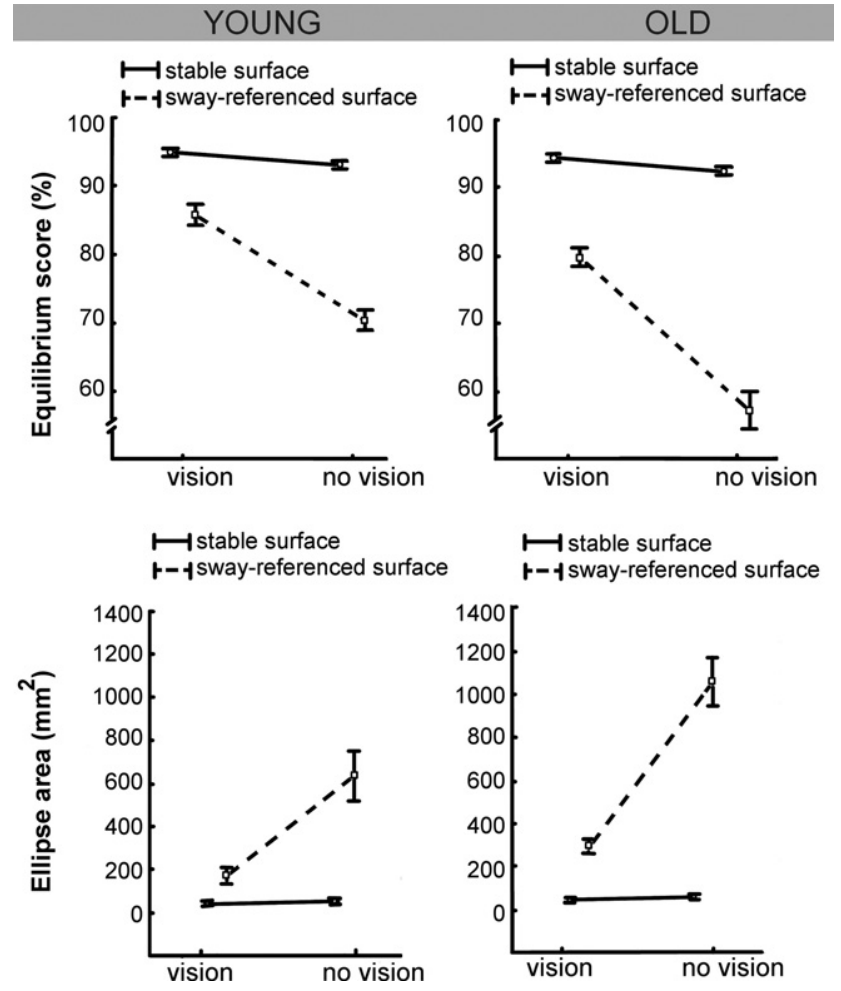

Fig. 2. Balance measures. Data points represent means \pm standard error (SE). Graphs show a significant 3-way interaction for both equilibrium score (Eq) (top graphs) and ellipse area (bottom graphs).

interaction between proprioceptive and visual feedback was present in both age groups, it was much more pronounced in the elderly. Omission of vision alone (SOT-B) did not have a significant effect on balance.

\subsection{DTI data}

\subsubsection{Age-related white matter changes}

Widespread age-related decreases in FA were detected in white matter pathways connecting frontal, parietal, occipital, and subcortical areas (Fig. 3). More specifically, the older group had a lower white matter integrity in the frontal forceps, genu and body of the corpus callosum, cingulum, fornix, anterior thalamic radiation, inferior fronto-occipital fasciculus, superior and inferior longitudinal fasciculus, and occipital forceps. Fig. 4 shows an increased axial and radial diffusivity in old as compared with young subjects. The changes in radial diffusivity mostly complied with FA changes. Age-related increased axial diffusivity was most prominent in the fornix.

\subsubsection{Voxelwise TBSS regression approach}

In the young group no white matter regions were found for which the FA was related to balance performance. In the old group, after correcting for possible confounding effects of age, correlations between white matter integrity and Eq were only found for conditions where proprioceptive feedback (SOT-C) or proprioceptive and visual feedback were compromised
(SOT-D). In both conditions a significant positive linear trend ( $p<0.05$, TFCE-corrected) was found between Eq and FA of parts of the following tracts: frontal forceps, left optic tract, bilateral anterior thalamic radiation, superior corona radiata, cingulum, corpus callosum, bilateral inferior longitudinal fasciculus, inferior fronto-occipital fasciculus, and occipital forceps (see Fig. 5). For these tracts lower FA-values were thus associated with lower balance performance. A more stringent threshold correction ( $p<0.05, t=3.1$, clustermass corrected) was used to further determine the association between specific white matter regions and balance performance under different sensory conditions (SOT-C and SOT-D).

\subsubsection{SOT-C: sway-referenced platform}

The FA of a cluster in the genu of the corpus callosum, extending to the frontal forceps bilaterally, was predictive (adjusted $R^{2}=0.40, p<0.001$ ) of performance on SOT-C (Fig. 6). Closer inspection of the data showed that for this cluster, 1 older subject with low balance performance also had a mean FA-value of more than 3 SD from the group mean. Reanalysis of the data following removal of this subject revealed that still $21 \%$ (adjusted $R^{2}=0.21, p<0.01$ ) of the variance in performance on SOT-C could be explained by the FA of the ROI in the frontal forceps (Fig. 6).

Voxelwise analysis of the axial and radial diffusivity was subsequently performed to determine whether FA-Eq relations could be attributed to either axonal (Song et al., 2003) or myelin degeneration (Song et al., 2002, 2005). It was found that primarily higher radial diffusivity in the frontal forceps was associated with lower balance performance.

\subsubsection{SOT-D: sway-referenced platform and eyes closed}

The FA of 2 clusters in the bilateral occipital forceps were predictive (adjusted $R^{2}=0.42$ ) of performance on SOT-D (see Fig. 6). For axial diffusivity no white matter voxels were found to correlate with balance performance. For radial diffusivity a negative linear trend was observed between balance performance and a region in the left occipital forceps, albeit at a nonsignificant level $(p<0.1)$.

\section{Discussion}

Previous work has demonstrated age-related changes in brain gray (Good et al., 2001; Kalpouzos et al., 2009; Smith et al., 2007) and white matter structure (Abe et al., 2002; Barrick et al., 2010; Nusbaum et al., 2001; Sullivan and Pfefferbaum, 2006, 2007; Sullivan et al., 2001; Zhang et al., 2010). These changes may be associated with cognitive and motor declines as well as altered sensorimotor processing. With respect to balance, studies investigating the role of white matter integrity in age-related balance decline have reported conflicting results (Ryberg et al., 2007; Sullivan et al., 2001, 2010) (see Introduction). This may partly be due to the different clinical test batteries used to assess balance control. Although highly relevant in patient popu- 


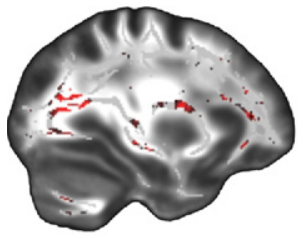

$-30$

X-coordinate $(\mathrm{mm})$

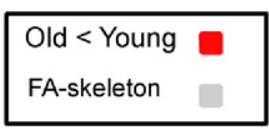

Z-coordinate $(\mathrm{mm})$

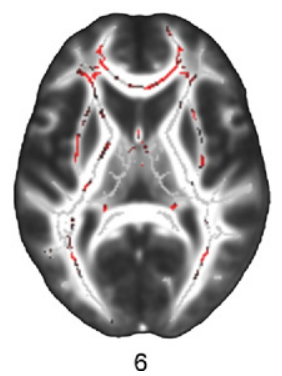

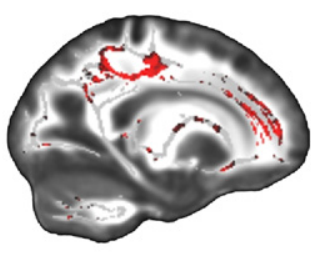

$-20$
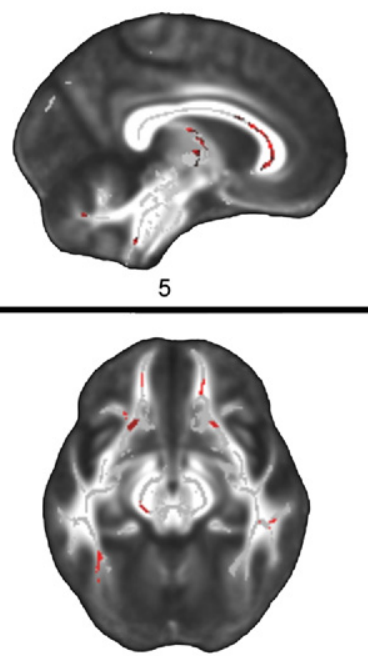

$-12$

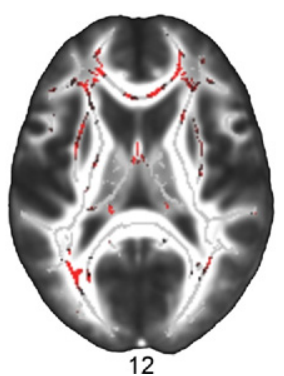

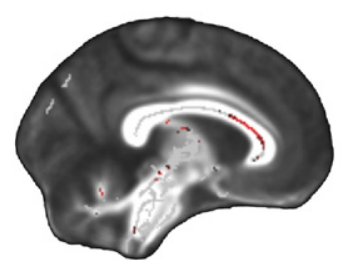

$-5$

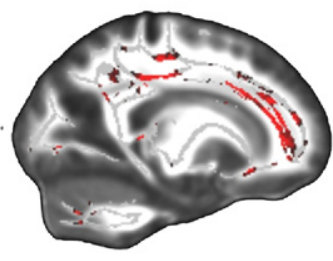

20

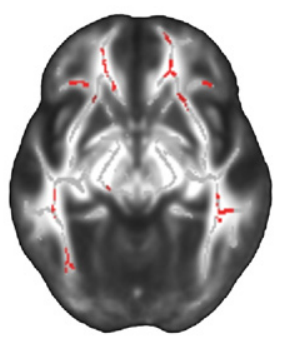

$-8$

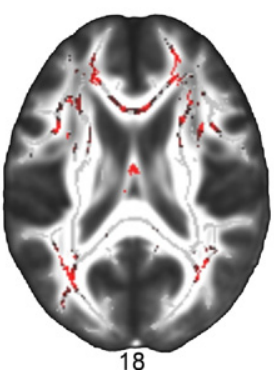

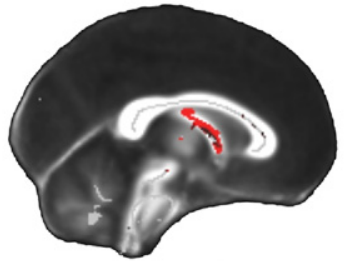

0
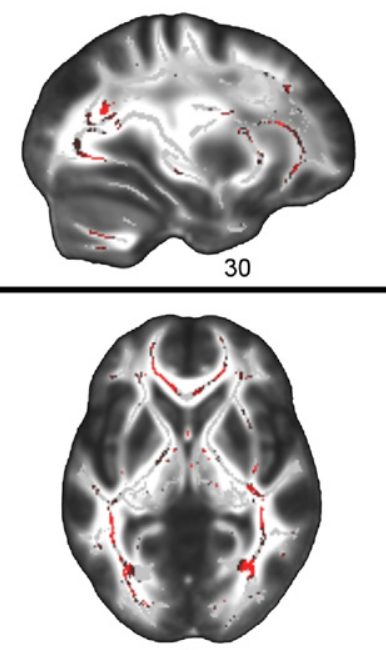

0

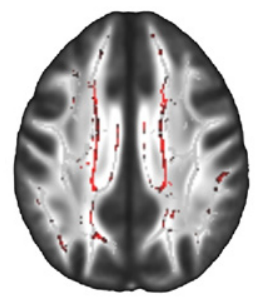

36

Fig. 3. Statistical maps of white matter voxels, having significantly ( $p<0.05, t=3.1$, cluster-mass corrected) smaller FA-values in the old than in the young group. Significant clusters are projected onto FMRIB58_FA_1-mm template in Montreal Neurological Institute (MNI) space, according to neurological convention.

lations, such tests may be less optimal to capture brain structure-balance relations in healthy elderly. Therefore in the current study, postural control was assessed by measuring body sway during upright stance on a dynamic balance platform. The applied SOT-protocol allowed the manipulation of visual and proprioceptive input. Consequently subjects were required to reweight sensory information to maintain balance, as is necessary during many daily life activities. TBSS was used to identify microstructural white matter changes that are predictive of postural control in aging. The results of this analysis will be discussed in more detail next.

\subsection{Age-related white matter degeneration}

In agreement with previous studies we observed agerelated FA decreases and radial diffusivity increases in the corpus callosum (Abe et al., 2002; Barrick et al., 2010;
Sullivan et al., 2001; Zahr et al., 2009; Zhang et al., 2008), cingulum (Barrick et al., 2010; Zahr et al., 2009), fornix (Zahr et al., 2009), inferior fronto-occipital fasciculus, (Hasan et al., 2010), and superior and inferior longitudinal fasciculus (Hasan et al., 2010; Zahr et al., 2009). Changes in radial diffusivity are often interpreted as reflecting a degeneration of myelin sheaths and/or an accumulation of glial cells (Song et al., 2002, 2005). In the elderly this is usually a consequence of ischemic changes (Baltan, 2009). FA decline may also be indirectly associated with reduced gray matter thickness, because both are correlated $(r=0.57)$ in the aged (Kochunov et al., 2007).

\subsection{The role of age-related white matter degeneration in postural control}

In agreement with previous studies using sway-referencing (Cohen et al., 1996; Doumas et al., 2008) or tendon 


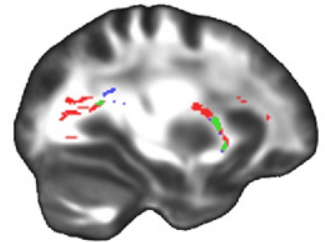

$-30$

X-coordinate $(\mathrm{mm})$

\begin{tabular}{|l|}
\hline Old>Young \\
$\mathrm{RD}$ \\
$\mathrm{AD}$ \\
$\mathrm{RD} \& \mathrm{AD}$ \\
\hline
\end{tabular}

Z-coordinate $(\mathrm{mm})$

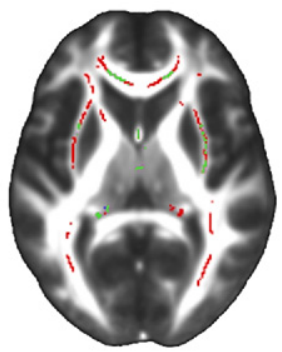

6

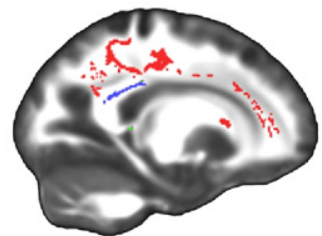

$-20$

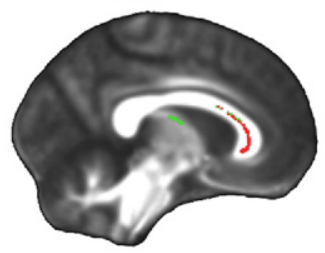

5

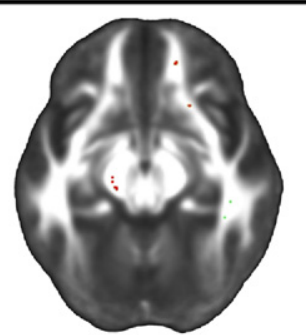

$-12$

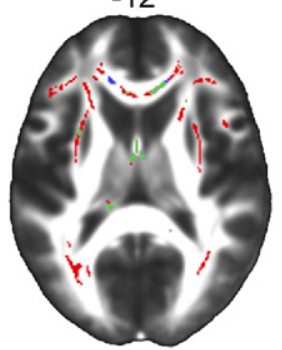

12

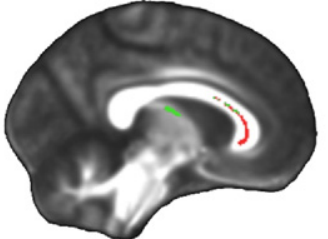

$-5$

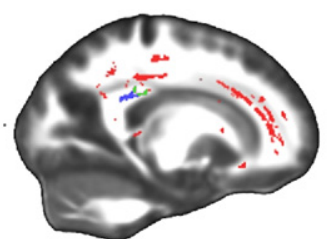

20

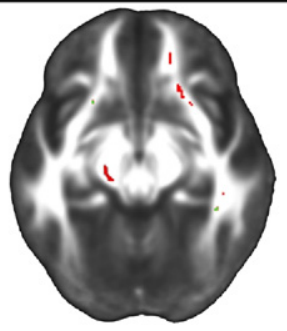

$-8$

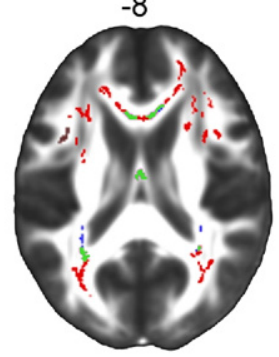

18

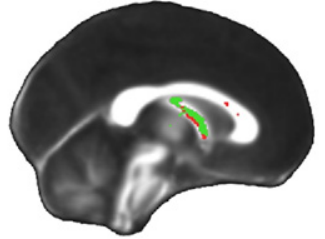

0

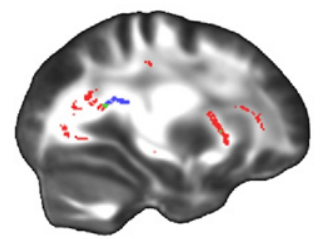

30
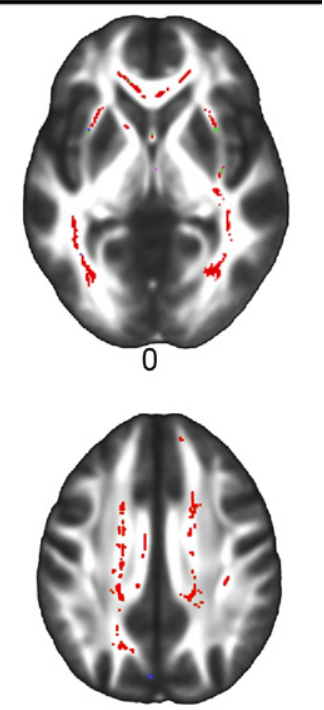

36

Fig. 4. Statistical maps of white matter voxels, having significantly ( $p<0.05, t=3.1$, cluster-mass corrected) higher axial (green and blue) and radial diffusivity (green and red) in the old than in the young group. Significant clusters are projected onto the FMRIB58_FA_1-mm template in Montreal Neurological Institute (MNI) space, according to neurological convention.

vibration (Speers et al., 2002; Teasdale and Simoneau, 2001) to manipulate proprioceptive feedback, we showed that both old and young subjects relied more on proprioception than on vision to maintain balance. More specifically, during upright stance on a stable surface (SOT-A) balance did not deteriorate when closing the eyes (SOT-B), whereas it did deteriorate when proprioceptive feedback was compromised, i.e., when the surface was sway-referenced (SOT-C). This effect was most pronounced in the older group. Teasdale and Simoneau (2001) demonstrated that elderly subjects also had more difficulty with reintegrating correct proprioceptive information, after proprioceptive inputs had been manipulated. They speculated that the elderly's difficulty in weighting sensory information to maintain balance could in part be explained by the reduced availability of brain resources. In agreement with their result we assumed that the requirement to reweight sensory inputs would allow the detection of meaningful brain structurebalance relations.

The force platform used in the present study manipulated ankle proprioceptive input under eyes open (SOT-C) and eyes closed conditions (SOT-D) (see 2.2.1. Methods). For both conditions various white matter clusters were observed for which FA showed a positive linear trend with balance performance. These clusters were situated in white matter tracts that were all affected by aging and involved afferent and efferent pathways, i.e., frontal forceps, left optic tract, bilateral anterior thalamic radiation, superior corona radiata, cingulum, corpus callosum, bilateral inferior longitudinal fasciculus, inferior fronto-occipital fasciculus, and occipital forceps. The role of these white matter tracts in balance performance is not surprising. Previous research has shown the involvement of the superior and middle frontal gyri, precentral gyrus, and superior pari- 


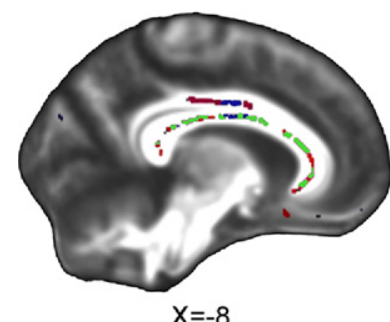

$X=-8$
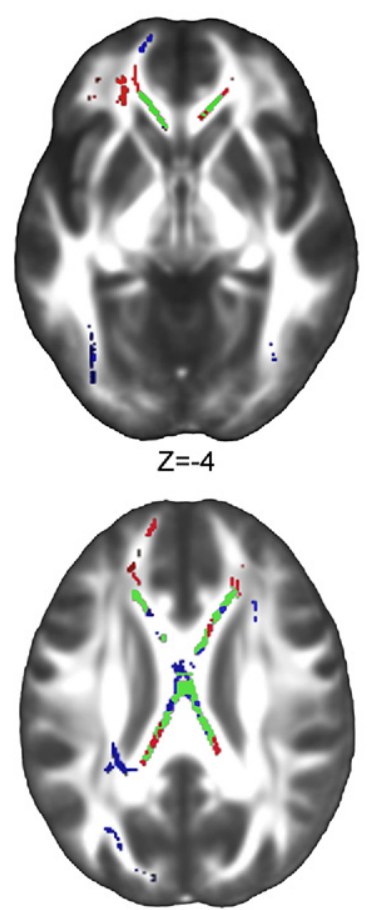

$Z=25$

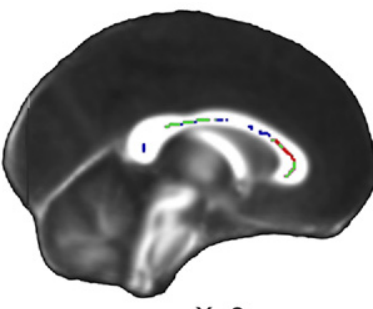

$\mathrm{X}=0$

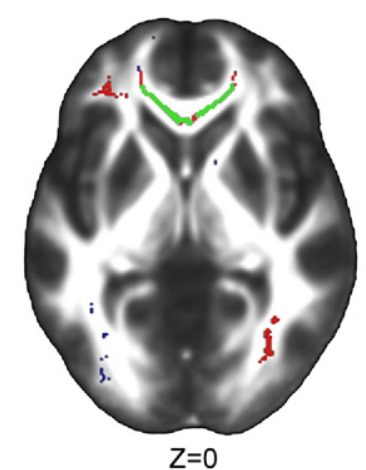

$\mathrm{Z}=0$

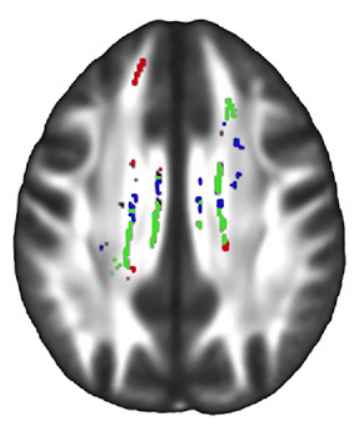

$Z=35$
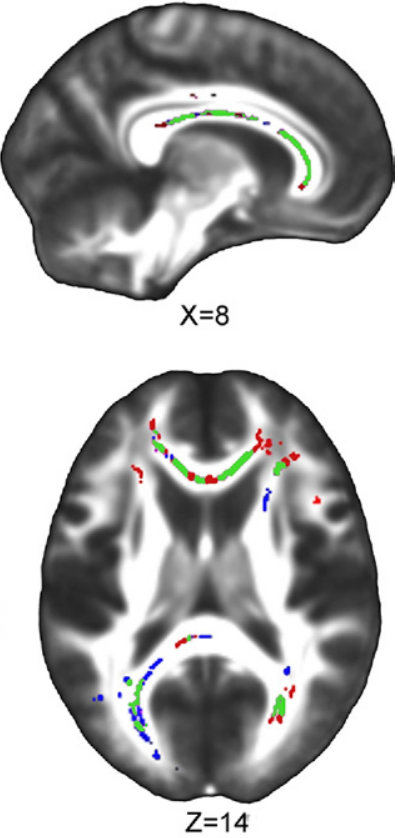

SOT-C

SOT-D

SOT-C \& SOT-D

Fig. 5. Statistical maps of white matter voxels, showing a significant ( $p<0.05$, threshold-free cluster enhancement [TFCE] corrected) linear trend between fractional anisotropy (FA)-values and equilibrium score (Eq) for Sensory Organization Test (SOT)-C and SOT-D, projected onto the FMRIB58_FA_1-mm template, according to neurological convention.

etal lobe in postural control (Mihara et al., 2008; Slobounov et al., 2005), thereby confirming that postural control is a complex sensorimotor coordination task that requires integration of different types of sensory information, and execution of corrective motor commands. This is enabled by transmission of information across cortical-subcortical pathways.

Since these structure-balance relations were not present in the young group, we assume that age-related white matter degeneration affects efficient transmission and processing of sensorimotor information, thereby contributing to balance impairments in the elderly. This implies that agerelated decline of postural control is dependent on multiple factors not only involving peripheral neural and musculoskeletal changes as well as altered postural strategies, but also changes in the central nervous system pathways. The effect of the various balance manipulations in the elderly is discussed next.

\subsubsection{Postural control in the elderly during manipulation of ankle proprioception}

Although various white matter tracts were correlated with balance performance in SOT-C (see 4.2. Discussion), i.e., when ankle proprioceptive feedback was compromised, more stringent thresholding showed that the integrity of a cluster in the genu of the corpus callosum alone explained $21 \%$ of the variance in balance performance. Further analysis revealed that this effect was more associated with radial as compared with axial diffusivity. Assuming that radial diffusivity is an indirect marker of myelin status (Song et al., 2002, 2005), the present data suggest that higher myelin degeneration of this region was associated with weaker balance performance.

The results are in accordance with a recent study showing that in homebound elderly suffering from various neurological diseases, white matter degeneration of an ROI in the genu of the corpus callosum was associated with gait 

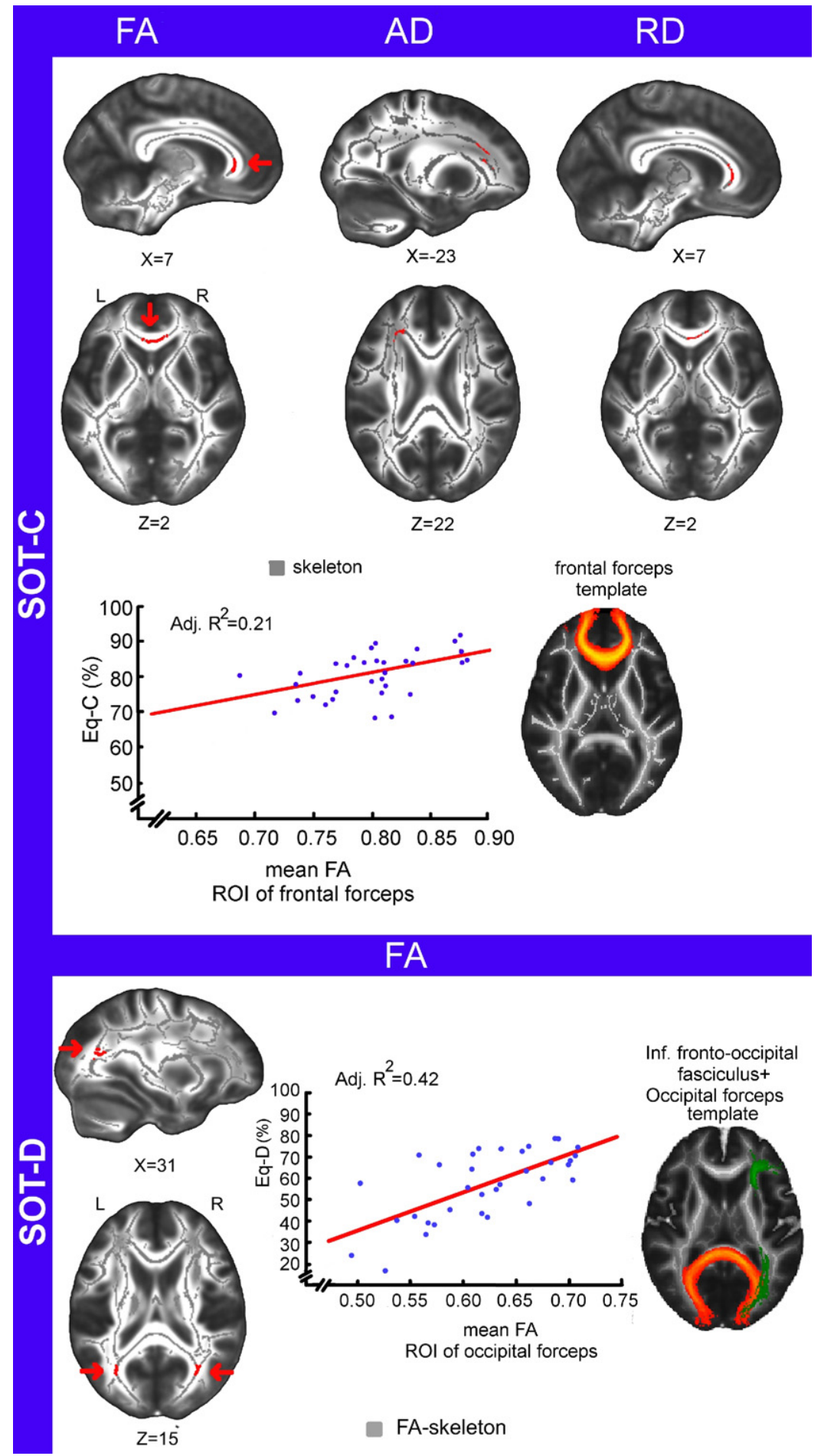

Fig. 6. Statistical maps of white matter voxels, showing a significant ( $p<0.05, t=3.1$, cluster-mass corrected) positive (fractional anisotropy; FA) or negative axial and radial diffusivity linear trend between white matter measures of the old group and equilibrium score (Eq), projected onto the FMRIB58_FA_1-mm template, according to neurological convention. The graph shows the result of a stepwise regression including age and the mean FA of an ROI (indicated by the red arrows) as predictors for balance performance during SOT-C (top graph) and SOT-D (bottom graph). L, left hemisphere; R, right hemisphere.

and balance impairment (Bhadelia et al., 2009). Our findings extend these results by showing that also in healthy older individuals the integrity of this area, as well as the projections to the frontopolar cortex (Park et al., 2008) may be associated with quality of postural control. The frontopolar cortex is involved in top-down control over actions and attentional shifts (Ramnani and Owen, 2004), as well as motor skill learning (Taubert et al., 2010). In the context of 
aging, frontal white matter tracts are of special interest since several research groups have put forth the so-called "frontal aging" hypothesis (Greenwood, 2000; West, 1996), postulating that aging affects anterior white matter tracts more than posterior ones. Thus tasks that require executive function or cognitive control over actions (Miller, 2000) would be more prone to age-related deficits. It is reasonable to assume that elderly make use of top-down mechanisms, exerting cognitive control over the motor system, to maintain balance, especially when proprioceptive feedback is compromised. More generally, elderly make increasingly use of cognitive resources to control action (Heuninckx et al., 2005, 2008; Swinnen et al., 2010). Various dual-task studies are also consistent with this idea because healthy elderly increasingly rely on cognitive reserve to safeguard their posture under attentionally more demanding balance conditions (Dault et al., 2001a, 2001b; Doumas et al., 2009; Huxhold et al., 2006; Rankin et al., 2000). At the same time, studies in patients with Alzheimer's disease (MazoterasMuñoz et al., 2010) show that they are more frequently prone to balance and gait impairment than are cognitively healthy elderly.

\subsubsection{Postural control in the elderly when ankle proprioception is manipulated and vision is omitted}

In SOT-D ankle proprioceptive feedback was compromised and visual feedback was absent, resulting in a higher reliance on the vestibular system for provision of correct postural information. Cortical (Emri et al., 2003; Fasold et al., 2002) and galvanic (Stephan et al., 2005) stimulations of the vestibular system have shown activations in a widespread vestibular cortical network comprising the temporoparietal junction, the insular cortex, the inferior frontal gyrus, and anterior cingulate gyrus. Furthermore vestibular stimulation leads to deactivations in the visual and somatosensory cortex as opposed to baseline (Stephan et al., 2005). This suggests that reciprocal inhibitory interactions exist between the vestibular network and the visual cortex (Brandt et al., 1998), that are present even when subjects are standing in complete darkness (Naito et al., 2003). Our observations also suggested the involvement of white matter pathways transmitting visual input under eyes-closed conditions. More specifically, bilateral areas in the occipital forceps predicted $42 \%$ of the variance in the older group's balance scores when only the vestibular system provided accurate feedback (SOT-D). The observed areas were situated in a region where occipital forceps and inferior frontooccipital fasciculus coincide. Among other things, these white matter tracts may play a role in the interactions between the vestibular and the visual system, described previously. The occipital forceps forms an interhemispheric connection between visual areas, whereas the inferior fronto-occipital fasciculus connects the dorsal prefrontal areas to posterior parietal and occipital areas (Catani et al., 2002; Doricchi et al., 2008; Martino et al., 2010). These tracts are important in the integration and reweighing of visual and proprioceptive information.

Even though referring to tracts involved in transmission of visual information in a condition deprived of visual input appears counterintuitive, various factors may contribute to this association. For example, it is conceivable that old subjects used some form of imagery to maintain their posture under eyes-closed conditions. Moreover, the role of the visual cortex may be more pronounced in the elderly due to an overall reduced inhibition in sensory cortices. For example, Zwergal et al. (2010) measured blood oxygen leveldependent (BOLD)-responses in old and young subjects while they imagined themselves standing. Although all subjects had their eyes closed, old subjects had higher BOLDresponses in vestibular as well as visual cortices (MT/V5).

Because the integrity of these tracts was not significantly correlated with SOT-B (stable surface, eyes closed) we assume that balance conditions have to be sufficiently challenging, i.e., ankle proprioceptive feedback is less reliable and visual cues cannot be used for orientation, for significant white matter integrity-balance relations to appear. Further research combining various brain imaging techniques will be necessary to further unravel the role of brain integrity and function in age-related balance impairments.

Another note of caution is that the prominence of tracts interconnecting cortical brain areas for predicting balance should not be taken to imply that corticocerebellar tracts, or vestibular pathways to the thalamus are of minor importance for postural control. Postural control is an extremely complex function that requires the intensive cooperation of cortical and subcortical structures. Nevertheless, the emergence of cortical pathways in predicting balance performance is consistent with the general notion that aging is associated with increased cognitive control over action (Heuninckx et al., 2005, 2008; Swinnen et al., 2010).

\subsection{Conclusions}

This is the first study, combining a voxel-based analysis of the white matter with dynamic posturography in the context of aging. Our results show that after removal of variance accounted for by age, white matter integrity of frontal and fronto-occipital tracts were predictive of balance performance in the older but not the younger group. These brain structure-balance relations were most pronounced when sensory feedback was manipulated. DTI may thus serve as a diagnostic tool for early identification of balance deficits in healthy elderly, as sensory systems start to decline. Therefore, therapies aimed at the improvement of balance performance should not only address musculoskeletal changes, but also try to stimulate neural processing by training subjects' postural control under conditions of manipulated sensory feedback, and making postural tasks more cognitively challenging. 


\section{Disclosure statement}

All authors declare there are no actual or potential conflicts of interest.

Participants were informed about the experimental procedures and provided written informed consent. The study was approved by the local Ethics Committee of K.U. Leuven and was performed in accordance with the 1964 Declaration of Helsinki.

\section{Acknowledgements}

Support for this study was provided through a grant from the Research Council of K.U. Leuven, Belgium (Contract OT/07/73), the Research Programme of the Research Foundation, Flanders (FWO, \# G.0593.08 and G.0483.10N), and Grant P6/29 from the Interuniversity Attraction Poles program of the Belgian federal government.

\section{References}

Abe, O., Aoki, S., Hayashi, N., Yamada, H., Kunimatsu, A., Mori, H., Yoshikawa, T., Okubo, T., Ohtomo, K., 2002. Normal aging in the central nervous system: quantitative MR diffusion-tensor analysis. Neurobiol. Aging 23, 433-441.

Aydoğ, S.T., Korkusuz, P., Doral, M.N., Tetik, O., Demirel, H.A., 2006. Decrease in the numbers of mechanoreceptors in rabbit ACL: the effects of ageing. Knee Surg. Sports Traumatol. Arthrosc. 14, 325-329.

Baltan, S., 2009. Ischemic injury to white matter: an age-dependent process. Neuroscientist 15, 126-133.

Barrick, T.R., Charlton, R.A., Clark, C.A., Markus, H.S., 2010. White matter structural decline in normal ageing: a prospective longitudinal study using tract-based spatial statistics. Neuroimage 51, 565577.

Bhadelia, R.A., Price, L.L., Tedesco, K.L., Scott, T., Qiu, W.Q., Patz, S., Folstein, M., Rosenberg, I., Caplan, L.R., Bergethon, P., 2009. Diffusion tensor imaging, white matter lesions, the corpus callosum, and gait in the elderly. Stroke 40, 3816-3820.

Black, F.O., Wall, C., III, Nashner, L.M., 1983. Effects of visual and support surface orientation references upon postural control in vestibular deficient subjects. Acta Otolaryngol. 95, 199-201.

Brandt, T., Bartenstein, P., Janek, A., Dieterich, M., 1998. Reciprocal inhibitory visual-vestibular interaction. Visual motion stimulation deactivates the parieto-insular vestibular cortex. Brain 121, 17491758.

Brouwer, B., Culham, E.G., Liston, R.A., Grant, T., 1998. Normal variability of postural measures: implications for the reliability of relative balance performance outcomes. Scand. J. Rehabil. Med. 30, 131-137.

Catani, M., Howard, R.J., Pajevic, S., Jones, D.K., 2002. Virtual in vivo interactive dissection of white matter fasciculi in the human brain. Neuroimage 17, 77-94.

Cohen, H., Heaton, L.G., Congdon, S.L., Jenkins, H.A., 1996. Changes in sensory organization test scores with age. Age Ageing 25, 39-44.

Dault, M.C., Frank, J.S., Allard, F., 2001a. Influence of a visuo-spatial, verbal and central executive working memory task on postural control. Gait Posture 14, 110-116.

Dault, M.C., Geurts, A.C., Mulder, T.W., Duysens, J., 2001b. Postural control and cognitive task performance in healthy participants while balancing on different support-surface configurations. Gait Posture 14, $248-255$.
Doricchi, F., Thiebaut de Schotten, M., Tomaiuolo, F., Bartolomeo, P., 2008. White matter (dis)connections and gray matter (dys)functions in visual neglect: gaining insights into the brain networks of spatial awareness. Cortex 44, 983-995.

Doumas, M., Krampe, R.T., 2010. Adaptation and reintegration of proprioceptive information in young and older adults' postural control. J. Neurophysiol. 104, 1969-1977.

Doumas, M., Rapp, M.A., Krampe, R.T., 2009. Working memory and postural control: adult age differences in potential for improvement, task priority, and dual tasking. J. Gerontol. B Psychol. Sci. Soc. Sci. 64, 193-201.

Doumas, M., Smolders, C., Krampe, R.T., 2008. Task prioritization in aging: effects of sensory information on concurrent posture and memory performance. Exp. Brain Res. 187, 275-281.

Duarte, M., Zatsiorsky, V.M., 2002. Effects of body lean and visual information on the equilibrium maintenance during stance. Exp. Brain Res. 146, 60-69.

Emri, M., Kisely, M., Lengyel, Z., Balkay, L., Márián, T., Mikó, L., Berényi, E., Sziklai, I., Trón, L., Tóth, A., 2003. Cortical projection of peripheral vestibular signaling. J. Neurophysiol. 89, 2639-2646.

Fasold, O., von Brevern, M., Kuhberg, M., Ploner, C.J., Villringer, A., Lempert, T., Wenzel, R., 2002. Human vestibular cortex as identified with caloric stimulation in functional magnetic resonance imaging. Neuroimage 17, 1384-1393.

Fife, T.D., Baloh, R.W. 1993. Disequilibrium of unknown cause in older people. Ann. Neurol. 34. 694-702.

Folstein, M.F., Folstein, S.E., McHugh, P.R., 1975. "Mini-mental state". A practical method for grading the cognitive state of patients for the clinician. J. Psychiatr. Res. 12, 189-198.

Goble, D.J., Coxon, J.P., Wenderoth, N., Van Impe, A., Swinnen, S.P., 2009. Proprioceptive sensibility in the elderly: Degeneration, functional consequences and plastic-adaptive processes. Neurosci. Biobehav. Rev. 33, 271-278.

Goebel, J.A., Sataloff, R.T., Hanson, J.M., Nashner, L.M., Hirshout, D.S., Sokolow, C.C., 1997. Posturographic evidence of nonorganic sway patterns in normal subjects, patients, and suspected malingerers. Otolaryngol. Head Neck Surg. 117, 293-302.

Good, C.D., Johnsrude, I.S., Ashburner, J., Henson, R.N., Friston, K.J., Frackowiak, R.S., 2001. A voxel-based morphometric study of ageing in 465 normal adult human brains. Neuroimage 14, 21-36.

Greenwood, P.M., 2000. The frontal aging hypothesis evaluated. J. Int. Neuropsychol. Soc. 6, 705-726.

Guttmann, C.R., Benson, R., Warfield, S.K., Wei, X., Anderson, M.C., Hall, C.B., Abu-Hasaballah, K., Mugler, J.P., III, Wolfson, L., 2000. White matter abnormalities in mobility-impaired older persons. Neurology 54, 1277-1283.

Hasan, K.M., Kamali, A., Abid, H., Kramer, L.A., Fletcher, J.M., EwingCobbs, L., 2010. Quantification of the spatiotemporal microstructural organization of the human brain association, projection and commissural pathways across the lifespan using diffusion tensor tractography. Brain Struct. Funct. 214, 361-373.

Heuninckx, S., Wenderoth, N., Debaere, F., Peeters, R., Swinnen, S.P., 2005. Neural basis of aging: the penetration of cognition into action control. J. Neurosci. 25, 6787-6796.

Heuninckx, S., Wenderoth, N., Swinnen, S.P., 2008. Systems neuroplasticity in the aging brain: recruiting additional neural resources for successful motor performance in elderly persons. J. Neurosci. 28, 91-99.

Horak, F.B., Shupert, C.L., Mirka, A., 1989. Components of postural dyscontrol in the elderly: a review. Neurobiol. Aging 10, 727-738.

Huxhold, O., Li, S.C., Schmiedek, F., Lindenberger, U., 2006. Dualtasking postural control: aging and the effects of cognitive demand in conjunction with focus of attention. Brain Res. Bull. 69, 294-305.

Jones, D.K., Basser, P.J., 2004. "Squashing peanuts and smashing pumpkins": how noise distorts diffusion-weighted MR data. Magn. Reson. Med. 52, 979-993. 
Kalpouzos, G., Chételat, G., Baron, J.C., Landeau, B., Mevel, K., Godeau, C., Barré, L., Constans, J.M., Viader, F., Eustache, F., Desgranges, B., 2009. Voxel-based mapping of brain gray matter volume and glucose metabolism profiles in normal aging. Neurobiol. Aging 30, 112-124.

Kochunov, P., Thompson, P.M., Lancaster, J.L., Bartzokis, G., Smith, S., Coyle, T., Royall, D.R., Laird, A., Fox, P.T., 2007. Relationship between white matter fractional anisotropy and other indices of cerebral health in normal aging: tract-based spatial statistics study of aging. Neuroimage 35, 478-487.

Leibowitz, H.M., Krueger, D.E., Maunder, L.R., Milton, R.C., Kini, M.M., Kahn, H.A., Nickerson, R.J., Pool, J., Colton, T.L., Ganley, J.P., Loewenstein, J.I., Dawber, T.R., 1980. The Framingham Eye Study monograph: An ophthalmological and epidemiological study of cataract, glaucoma, diabetic retinopathy, macular degeneration, and visual acuity in a general population of 2631 adults, 1973-1975. Surv. Ophthalmol. 24, 335-610.

Lord, S.R., Clark, R.D., Webster, I.W., 1991. Physiological factors associated with falls in an elderly population. J. Am. Geriatr. Soc. 39, 1194-1200.

Maki, B.E., Perry, S.D., Norrie, R.G., McIlroy, W.E., 1999. Effect of facilitation of sensation from plantar foot-surface boundaries on postural stabilization in young and older adults. J. Gerontol. A Biol. Sci. Med. Sci. 54, M281-M287.

Martino, J., Brogna, C., Robles, S.G., Vergani, F., Duffau, H., 2010. Anatomic dissection of the inferior fronto-occipital fasciculus revisited in the lights of brain stimulation data. Cortex 46, 691-699.

Mazoteras Muñoz, V., Abellan van Kan, G., Cantet, C., Cortes, F., Ousset, P.J., Rolland, Y., Vellas, B., 2010. Gait and balance impairments in Alzheimer disease patients. Alzheimer Dis. Assoc. Disord. 24, 79-84.

Mihara, M., Miyai, I., Hatakenaka, M., Kubota, K., Sakoda, S., 2008. Role of the prefrontal cortex in human balance control. Neuroimage 43, 329-336.

Miller, E.K., 2000. The prefrontal cortex and cognitive control. Nat. Rev. Neurosci. 1, 59-65.

Monsell, E.M., Furman, J.M., Herdman, S.J., Konrad, H.R., Shepard, N.T., 1997. Technology Assessment: Computerized dynamic platform posturography. Otolarynogol. Head Neck Surg. 117, 394-398.

Naito, Y., Tateya, I., Hirano, S., Inoue, M., Funabiki, K., Toyoda, H., Ueno, M., Ishizu, K., Nagahama, Y., Fukuyama, H., Ito, J., 2003. Cortical correlates of vestibulo-ocular reflex modulation: a PET study. Brain 126, 1562-1578.

Nashner, L.M., Black, F.O., Wall, C., III, 1982. Adaptation to altered support and visual conditions during stance: patients with vestibular deficits. J. Neurosci. 2, 536-544.

Nichols, T.E., Holmes, A.P., 2002. Nonparametric permutation tests for functional neuroimaging: a primer with examples. Hum. Brain Mapp. $15,1-25$.

Nusbaum, A.O., Tang, C.Y., Buchsbaum, M.S., Wei, T.C., Atlas, S.W., 2001. Regional and global changes in cerebral diffusion with normal aging. AJNR Am. J. Neuroradiol. 22, 136-142.

Oldfield, R.C., 1971. The assessment and analysis of handedness: the Edinburgh inventory. Neuropsychologia 9, 97-113.

Oliveira, L.F., Simpson, D.M., Nadal, J., 1996. Calculation of area of stabilometric signals using principal component analysis. Physiol. Meas. 17, 305-312.

Park, H.J., Kim, J.J., Lee, S.K., Seok, J.H., Chun, J., Kim, D.I., Lee, J.D., 2008. Corpus callosal connection mapping using cortical gray matter parcellation and DT-MRI. Hum. Brain Mapp. 29, 503-516.

Ramnani, N., Owen, A.M., 2004. Anterior prefrontal cortex: insights into function from anatomy and neuroimaging. Nat. Rev. Neurosci. 5, 184-194.

Rankin, J.K., Woollacott, M.H., Shumway-Cook, A., Brown, L.A., 2000. Cognitive influence on postural stability: a neuromuscular analysis in young and older adults. J. Gerontol. A Biol. Sci. Med. Sci. 55, M112M119.
Ryberg, C., Rostrup, E., Stegmann, M.B., Barkhof, F., Scheltens, P., van Straaten, E.C., Fazekas, F., Schmidt, R., Ferro, J.M., Baezner, H., Erkinjuntti, T., Jokinen, H., Wahlund, L.O., O’Brien, J., Basile, A.M., Pantoni, L., Inzitari, D., Waldemar, G., LADIS study group, 2007. Clinical significance of corpus callosum atrophy in a mixed elderly population. Neurobiol. Aging 28, 955-963.

Slobounov, S., Hallett, M., Stanhope, S., Shibasaki, H., 2005. Role of cerebral cortex in human postural control: an EEG study. Clin. Neurophysiol. 116, 315-323.

Smith, C.D., Chebrolu, H., Wekstein, D.R., Schmitt, F.A., Markesbery, W.R., 2007. Age and gender effects on human brain anatomy: a voxel-based morphometric study in healthy elderly. Neurobiol. Aging 28, 1075-1087.

Smith, S.M., Jenkinson, M., Johansen-Berg, H., Rueckert, D., Nichols, T.E., Mackay, C.E., Watkins, K.E., Ciccarelli, O., Cader, M.Z., Matthews, P.M., Behrens, T.E., 2006. Tract-based spatial statistics: voxelwise analysis of multi-subject diffusion data. Neuroimage 31, 14871505.

Smith, S.M., Jenkinson, M., Woolrich, M.W., Beckmann, C.F., Behrens, T.E., Johansen-Berg, H., Bannister, P.R., De Luca, M., Drobnjak, I., Flitney, D.E., Niazy, R.K., Saunders, J., Vickers, J., Zhang, Y., De Stefano, N., Brady, J.M., Matthews, P.M., 2004. Advances in functional and structural MR image analysis and implementation as FSL. Neuroimage 23, S208-S219.

Smith, S.M., Nichols, T.E., 2009. Threshold-free cluster enhancement: addressing problems of smoothing, threshold dependence and localisation in cluster inference. Neuroimage 44, 83-98.

Song, S.K., Sun, S.W., Ju, W.K., Lin, S.J., Cross, A.H., Neufeld, A.H., 2003. Diffusion tensor imaging detects and differentiates axon and myelin degeneration in mouse optic nerve after retinal ischemia. Neuroimage 20, 1714-1722.

Song, S.K., Sun, S.W., Ramsbottom, M.J., Chang, C., Russell, J., Cross, A.H., 2002. Dysmyelination revealed through MRI as increased radial (but unchanged axial) diffusion of water. Neuroimage 17, $1429-1436$.

Song, S.K., Yoshino, J., Le, T.Q., Lin, S.J., Sun, S.W., Cross, A.H., Armstrong, R.C., 2005. Demyelination increases radial diffusivity in corpus callosum of mouse brain. Neuroimage 26, 132-140.

Speers, R.A., Kuo, A.D., Horak, F.B., 2002. Contributions of altered sensation and feedback responses to changes in coordination of postural control due to aging. Gait Posture 16, 20-30.

Stelmach, G.E., Teasdale, N., Di Fabio, R.P., Phillips, J., 1989. Age related decline in postural control mechanisms. Int. J. Aging Hum. Dev. 29, 205-223.

Stephan, T., Deutschländer, A., Nolte, A., Schneider, E., Wiesmann, M., Brandt, T., Dieterich, M., 2005. Functional MRI of galvanic vestibular stimulation with alternating currents at different frequencies. Neuroimage 26, 721-732.

Sullivan, E.V., Adalsteinsson, E., Hedehus, M., Ju, C., Moseley, M., Lim, K.O., Pfefferbaum, A., 2001. Equivalent disruption of regional white matter microstructure in ageing healthy men and women. Neuroreport $12,99-104$.

Sullivan, E.V., Pfefferbaum, A., 2006. Diffusion tensor imaging and aging. Neurosci. Biobehav. Rev. 30, 749-761.

Sullivan, E.V., Pfefferbaum, A., 2007. Neuroradiological characterization of normal adult ageing. Br. J. Radiol. 80 Spec No 2, S99-S108.

Sullivan, E.V., Rohlfing, T., Pfefferbaum, A., 2010. Quantitative fiber tracking of lateral and interhemispheric white matter systems in normal aging: Relations to timed performance. Neurobiol. Aging $31,464-481$.

Sullivan, E.V., Rose, J., Rohlfing, T., Pfefferbaum, A., 2009. Postural sway reduction in aging men and women: Relation to brain structure, cognitive status, and stabilizing factors. Neurobiol. Aging 30, 793-807.

Swinnen, S.P., Heuninckx, S., Van Impe, A., Goble, D.J., Coxon, J.P., Wenderoth, N., 2010. Aging and movement control: the neural basis of age-related compensatory recruitment, in: Danion, F., Latash, M. 
(Eds.), Motor Control: Theories, Experiments, and Applications. Oxford University Press, New York, pp. 383-413.

Taubert, M., Draganski, B., Anwander, A., Müller, K., Horstmann, A., Villringer, A., Ragert, P., 2010. Dynamic properties of human brain structure: learning-related changes in cortical areas and associated fiber connections. J. Neurosci. 30, 11670-11677.

Teasdale, N., Simoneau, M., 2001. Attentional demands for postural control: the effects of aging and sensory reintegration. Gait Posture 14, 203-210.

West, R.L., 1996. An application of prefrontal cortex function theory to cognitive aging. Psychol. Bull. 120, 272-292.
Zahr, N.M., Rohlfing, T., Pfefferbaum, A., Sullivan, E.V., 2009. Problem solving, working memory, and motor correlates of association and commissural fiber bundles in normal aging: a quantitative fiber tracking study. Neuroimage 44, 1050-1062.

Zhang, Y., Du, A.T., Hayasaka, S., Jahng, G.H., Hlavin, J., Zhan, W., Weiner, M.W., Schuff, N., 2010. Patterns of age-related water diffusion changes in human brain by concordance and discordance analysis. Neurobiol. Aging 31, 1991-2001.

Zwergal, A., Linn, J., Xiong, G., Brandt, T., Strupp, M., Jahn, K., 2010. Aging of human supraspinal locomotor and postural control in fMRI. Neurobiol. Aging, in press. doi: 10.1016/j.neurobiolaging.2010.09.022. 